\title{
Management of Myasthenia Gravis During COVID-19 Pandemic
}

\author{
Xingxing $\mathrm{Wu}^{1 \dagger}$, Jiang Yuan ${ }^{1 \dagger}$, Md Rezaul Karim ${ }^{2}$, Yun-Fu Wang ${ }^{{ }^{*}}$
}

${ }^{1}$ Dept. of Neurology, Taihe Hospital, Hubei University of Medicine, Shiyan, China.

${ }^{2}$ Hubei Key Laboratory of Embryonic Stem Cell Research, Institute of Neuroscience, Hubei University of Medicine, Shiyan, China.

†uthors contributed equally to this work.

*Correspondence to: Prof. Dr. Yun-Fu Wang, Ph.D., MD; Dept. of Neurology, Taihe Hospital, Hubei University of Medicine, Shiyan, Hubei, China; Email: wangyun@hbmu.edu.cn

\begin{abstract}
The outbreak of COVID-19 has brought unprecedented risks and challenges to everyone in the world. Myasthenia gravis is an autoimmune disease of the nervous system. Infection can worsen it and cause severe symptoms such as myasthenia crisis and respiratory failure. At present, the pandemic of COVID-19 may promote the aggravation of patients with MG. This article focuses on the respiratory management of MG patients during the epidemic of COVID-19.
\end{abstract}

KEYWORDS: COVID-19; myasthenia gravis; respiratory management

\section{INTRODUCTION}

In December 2019, Wuhan, China, reported a series of pneumonia cases caused by unknown causes ${ }^{1}$. The pathogen was quickly identified as a new coronavirus associated with SARS pathogen of severe acute respiratory syndrome and named SARS coronavirus-19 (SARS-CoV-2) ${ }^{2}$. The infection then spread rapidly around the world and was declared a pandemic by the World Health Organization, which was named coronavirus disease 2019 (COVID-19) ${ }^{3}$. COVID-19 virus is a newly discovered coronavirus with a size of $60-140 \mathrm{~nm}$, which is similar to SARS-CoV of severe acute respiratory syndrome and belongs to the $\beta$-coronavirus genus of Coronaviridae ${ }^{4-6}$. COVID-19 patients have a variety of symptoms, including fever, cough, respiratory symptoms, diarrhea, loss of smell and taste, severe acute respiratory distress syndrome, and even death ${ }^{7}$. Myasthenia gravis (MG) is a T cell-dependent 
autoimmune disease mediated by antibody complements, characterized by fatigue muscle weakness associated with acetylcholine receptor (ACHR), muscle-specific kinase (Musk), lipoprotein-associated protein 4 (LRP4) or postsynaptic membrane aggregin antibody of the neuromuscular junction $(\mathrm{NMJ})^{8}$. In the retrospective study, the disorder of immune regulation caused by infection has been the main cause of the deterioration of myasthenia gravis ${ }^{9}$. However, all patients with severe respiratory failure caused by COVID-19 had a strong inflammatory response to immune regulation disorder or macrophage activation syndrome (MAS) ${ }^{10}$. If MG patients are infected with COVID-19, their disease progression may be accelerated, or their symptoms may worsen. Therefore, it is particularly important to manage MG patients during the period of COVID-19 epidemic. Among them, controlling the symptoms of MG, delaying the progression of the disease, preventing the occurrence of myasthenia crisis, and doing proper respiratory management are the top priorities of the management of MG patients.

\section{COMPREHENSIVE MANAGEMENT OF MG DURING COVID-19 PANDEMIC}

The management of MG aims to relieve symptoms by controlling disease activities, monitoring treatment-related adverse events, and personalized support measures, so that the recovery of muscle function and the improvement of patients' quality of life are close to those of normal people ${ }^{11}$. Conventional treatments for MG include symptomatic treatment with acetylcholinesterase inhibitors, thymectomy, and immunotherapy. Intravenous immunoglobulin and plasma exchange is rapid treatments for myasthenia crisis and disease deterioration ${ }^{12,13}$. Mechanical ventilation and intensive care are needed for the deterioration of respiratory failure. For MG patients who are not infected with COVID-19, they can keep the original treatment unchanged and consult neurologists in time to adjust according to the degree of symptoms. At present, no specific drugs for COVID-19 have been found. MG patients who are not infected with COVID-19 should not participate in personnel gathering activities as much as possible. It is best to be isolated at home. When they have got to go out, pay attention to maintaining a social distance of more than 1 meter, and pay attention to frequent hand washing and hand hygiene.

At present, no data have been reported on the risk of deterioration of MG caused by 
COVID-19 or previous coronaviruses. If MG patients are complicated with COVID-19 infection, we recommend that the respiratory function of patients should be closely monitored. For patients with mild myasthenia gravis, appropriate cholinesterase inhibitors can be used to improve muscle function, contraction, and strength ${ }^{13}$. When the effect of a cholinesterase inhibitor is not obvious, immunotherapy can be used to improve the symptoms of myasthenia gravis. Corticosteroids such as prednisolone are the first choice of immunotherapy ${ }^{14}$. For patients with severe myasthenia gravis, Gamma globulin for intravenous injection and plasma exchange can be used, and thymectomy or thymic radiotherapy can be performed according to the actual situation of the patients.

\section{RESPIRATORY MANAGEMENT IN MG PATIENTS WITH COVID-19 INFECTION}

Novel coronavirus has a tendency towards the lungs and can cause community-acquired pneumonia (CAP). The most common symptoms include fever (98.6\%), fatigue $(69.6 \%)$, dry cough, and diarrhea ${ }^{15,16}$. Some patients with new coronary pneumonia suddenly deteriorate into severe respiratory failure(SRF), requires intubation and mechanical ventilation(MV), patients with respiratory failure have a high risk of death, even up to $60 \%{ }^{17}$. Respiratory failure caused by COVID-19 infection is mainly due to pulmonary interstitial damage caused by the virus, which leads to pulmonary ventilation dysfunction. Myasthenia crisis in patients with $\mathrm{MG}$ is due to the rapid deterioration of $\mathrm{MG}$, involving respiratory muscles and upper respiratory muscles, resulting in pulmonary ventilatory dysfunction, resulting in respiratory failure. Infection is a common inducing factor of myasthenia crisis ${ }^{18}$. When myasthenia crisis occurs in MG patients with COVID-19 infection, it may progress to more severe respiratory failure. Therefore, when MG patients are infected with COVID19, the respiratory function should be closely monitored to prevent the occurrence of myasthenia crisis and respiratory failure, which is the key to their respiratory management. In case of an emergency such as myasthenia crisis or respiratory failure, timely intubation, and mechanical ventilation is very important.

Studies have shown that early MG patients with any obvious symptoms of respiratory involvement should be sent to the ICU ward for continuous respiratory function monitoring. This includes changes in respiratory indexes such as forced vital capacity (FVC) and 
maximum inspiratory negative pressure (NIF) ${ }^{19}$. In the process of monitoring, non-invasive ventilation can be carried out as soon as possible according to the respiratory function of the patients. Early non-invasive ventilation can avoid the complications of mechanical ventilation in patients with myasthenia crisis ${ }^{22,23}$.If NIF $<-20 \mathrm{cmH}_{2} \mathrm{O}, \mathrm{FVC}<15 \mathrm{~mL} / \mathrm{kg}$ indicates that respiratory failure may occur, endotracheal intubation and mechanical ventilation can be started $^{20,21}$. Rabinstein $\mathrm{A}$ et $\mathrm{al}^{21}$ studies suggest that biphasic positive airway pressure ventilation(BiPAP) can prevent intubation in patients with myasthenia crisis without obvious hypercapnia, BiPAP can be used before the onset of hypercapnia $(\mathrm{PaCO} 2>45 \mathrm{mmHg})$. Severe hypercapnia $(\mathrm{PaCO} 2>45 \mathrm{mmHg})$, hypoxemia $(\mathrm{PaO} 2<60 \mathrm{mmHg})$ and serum bicarbonate concentration $<30 \mathrm{mmol} / \mathrm{L}$ may indicate the failure of non-invasive ventilation $^{23,24}$. Intubation and mechanical ventilation should be done as soon as possible. Once intubated, most MG patients will be mechanically ventilated for more than a week ${ }^{23,25,26}$. MG patients with COVID19 infection may maintain mechanical ventilation for a longer time.

Cholinesterase inhibitors should be temporarily stopped after intubation to prevent excessive airway secretions ${ }^{27}$. During mechanical ventilation, plasmapheresis or intravenous immunoglobulin can be used to prevent further progression of myasthenia gravis. Plasma exchange and intravenous immunoglobulin are thought to shorten the duration of mechanical ventilation and achieve good benefits in the short term ${ }^{28-30}$. In the process of mechanical ventilation, chest physiotherapy should be done well, and sputum suction should be done in time to prevent complications such as atelectasis and so on.

The timing of weaning and extubation should be managed individually according to the patient's condition. Studies have found that $25 \%$ to $40 \%$ of critically ill patients with MG cannot be extubated $23,24,31$. Weaning should not be attempted when forced vital capacity (FVC) and maximum inspiratory negative pressure (NIF) do not show signs of improvement in respiratory muscle strength. Cholinesterase inhibitors can be used before weaning $21,24,32$. Excessive secretions can be treated with small doses of glycyrrhetinic acid or anisodamine. BiPAP can be used after extubation to help prevent re-intubation ${ }^{21}$.

COVID-19 is highly contagious, so medical staff needs to pay attention to personal safety protection during the whole process of respiratory management (see Figure 1), especially 
during endotracheal intubation and mechanical ventilation.

\section{CONCLUSION}

MG is an autoimmune disease of the nervous system. Infection can easily aggravate the symptoms of myasthenia gravis and even lead to myasthenia crisis. The risk of critical illness such as myasthenia crisis and respiratory failure in MG patients with COVID-19 infection may increase. During the COVID-19 pandemic, MG patients should take good personal protection to avoid infection with the virus. When MG patients are infected with COVID19, we should pay attention to respiratory muscle involvement, closely monitor respiratory function, timely symptomatic treatment, and do a good job in respiratory management.

\section{CONSENT FOR PUBLICATION}

All the authors have agreed to publish this work.

\section{AUTHOR CONTRIBUTIONS}

XW and JY: concept, writing, and review of the manuscript, MRK: review and editing of the manuscript, and YFW: supervision and critical review of the manuscript.

\section{ACKNOWLEDGMENTS}

Authors thanks to the Institute of Neuroscience, Hubei University of Medicine, Shiyan, Hubei, China, for all the help.

\section{FUNDING}

National Natural Science Foundation of China (No. 81672138); Hubei university of medicine graduate science and technology innovation project (No. YC2019033).

\section{Uncategorized References}

1. Amodio, E., Vitale, F., Cimino, L., Casuccio, A. \& Tramuto, F. Outbreak of Novel Coronavirus (SARS-Cov-2): First Evidences From International Scientific Literature and Pending Questions. Healthcare (Basel, Switzerland) 8(2020).

2. Dilcher, M., Werno, A. \& Jennings, L.C. SARS-CoV-2: a novel deadly virus in a 
globalised world. The New Zealand medical journal 133, 6-11 (2020).

3. Hsu, L.Y., Chia, P.Y. \& Lim, J.F. The Novel Coronavirus (SARS-CoV-2) Epidemic. Annals of the Academy of Medicine, Singapore 49, 105-107 (2020).

4. Chen, L., et al. RNA based mNGS approach identifies a novel human coronavirus from two individual pneumonia cases in 2019 Wuhan outbreak. Emerging microbes \& infections 9, 313-319 (2020).

5. Lu, R., et al. Genomic characterisation and epidemiology of 2019 novel coronavirus: implications for virus origins and receptor binding. Lancet (London, England) 395, 565-574 (2020).

6. Paraskevis, D., et al. Full-genome evolutionary analysis of the novel corona virus (2019-nCoV) rejects the hypothesis of emergence as a result of a recent recombination event. Infection, genetics and evolution : journal of molecular epidemiology and evolutionary genetics in infectious diseases 79, 104212 (2020).

7. Jacob, S., et al. Guidance for the management of myasthenia gravis (MG) and Lambert-Eaton myasthenic syndrome (LEMS) during the COVID-19 pandemic. Journal of the neurological sciences 412, 116803 (2020).

8. Gilhus, N.E. \& Verschuuren, J.J. Myasthenia gravis: subgroup classification and therapeutic strategies. The Lancet. Neurology 14, 1023-1036 (2015).

9. Gummi, R.R., Kukulka, N.A., Deroche, C.B. \& Govindarajan, R. Factors associated with acute exacerbations of myasthenia gravis. Muscle \& nerve 60, 693-699 (2019).

10. Giamarellos-Bourboulis, E.J., et al. Complex Immune Dysregulation in COVID-19 Patients with Severe Respiratory Failure. Cell host \& microbe (2020).

11. Gilhus, N.E., et al. Myasthenia gravis. Nature reviews. Disease primers 5, 30 (2019).

12. Gajdos, P., Chevret, S., Clair, B., Tranchant, C. \& Chastang, C. Clinical trial of plasma exchange and high-dose intravenous immunoglobulin in myasthenia gravis. Myasthenia Gravis Clinical Study Group. Annals of neurology 41, 789-796 (1997).

13. Gajdos, P., Chevret, S. \& Toyka, K.V. Intravenous immunoglobulin for myasthenia gravis. The Cochrane database of systematic reviews 12, Cd002277 (2012).

14. Blum, S., et al. Use and monitoring of low dose rituximab in myasthenia gravis. Journal of neurology, neurosurgery, and psychiatry 82, 659-663 (2011). 
15. Cascella, M., Rajnik, M., Cuomo, A., Dulebohn, S.C. \& Di Napoli, R. Features, Evaluation and Treatment Coronavirus (COVID-19). in StatPearls (StatPearls Publishing Copyright (C) 2020, StatPearls Publishing LLC., Treasure Island (FL), 2020).

16. Wang, Y., Wang, Y., Chen, Y. \& Qin, Q. Unique epidemiological and clinical features of the emerging 2019 novel coronavirus pneumonia (COVID-19) implicate special control measures. Journal of medical virology (2020).

17. Arabi, Y.M., Murthy, S. \& Webb, S. Correction to: COVID-19: a novel coronavirus and a novel challenge for critical care. Intensive care medicine 46, 1087-1088 (2020).

18. Lizarraga, A.A., Lizarraga, K.J. \& Benatar, M. Getting Rid of Weakness in the ICU: An Updated Approach to the Acute Management of Myasthenia Gravis and Guillain-Barré Syndrome. Seminars in neurology 36, 615-624 (2016).

19. Rieder, P., Louis, M., Jolliet, P. \& Chevrolet, J.C. The repeated measurement of vital capacity is a poor predictor of the need for mechanical ventilation in myasthenia gravis. Intensive care medicine 21, 663-668 (1995).

20. Agarwal, R., Reddy, C. \& Gupta, D. Non-invasive ventilation in acute neuromuscular respiratory failure due to myasthenic crisis: case report and review of literature. Emergency medicine journal : EMJ 23, e6 (2006).

21. Rabinstein, A. \& Wijdicks, E.F. BiPAP in acute respiratory failure due to myasthenic crisis may prevent intubation. Neurology 59, 1647-1649 (2002).

22. Fernández, J.A., Fernández-Valiñas, A., Hernández, D., Orozco, J. \& Lugo, A. Myasthenic Crisis in an Elderly Patient with Positive Antibodies against Acetylcholine and Anti-MuSK, Successfully Treated with Noninvasive Mechanical Ventilation. Case reports in critical care 2015, 624718 (2015).

23. Seneviratne, J., Mandrekar, J., Wijdicks, E.F. \& Rabinstein, A.A. Non-invasive ventilation in myasthenic crisis. Archives of neurology 65, 54-58 (2008).

24. Wu, J.Y., et al. The role of non-invasive ventilation and factors predicting extubation outcome in myasthenic crisis. Neurocritical care 10, 35-42 (2009).

25. Kalita, J., Kohat, A.K. \& Misra, U.K. Predictors of outcome of myasthenic crisis. Neurological sciences : official journal of the Italian Neurological Society and of the 
Italian Society of Clinical Neurophysiology 35, 1109-1114 (2014).

26. Thomas, C.E., et al. Myasthenic crisis: clinical features, mortality, complications, and risk factors for prolonged intubation. Neurology 48, 1253-1260 (1997).

27. Juel, V.C. Myasthenia gravis: management of myasthenic crisis and perioperative care. Seminars in neurology 24, 75-81 (2004).

28. Dhawan, P.S., et al. IVIG Versus PLEX in the Treatment of Worsening Myasthenia Gravis: What is the Evidence?: A Critically Appraised Topic. The neurologist 19, 145-148 (2015).

29. Wendell, L.C. \& Levine, J.M. Myasthenic crisis. The Neurohospitalist 1, 16-22 (2011).

30. Neumann, B., et al. Myasthenic crisis demanding mechanical ventilation: A multicenter analysis of 250 cases. Neurology 94, e299-e313 (2020).

31. Rabinstein, A.A. \& Mueller-Kronast, N. Risk of extubation failure in patients with myasthenic crisis. Neurocritical care 3, 213-215 (2005).

32. Rabinstein, A.A. Acute Neuromuscular Respiratory Failure. Continuum (Minneapolis, Minn.) 21, 1324-1345 (2015). 


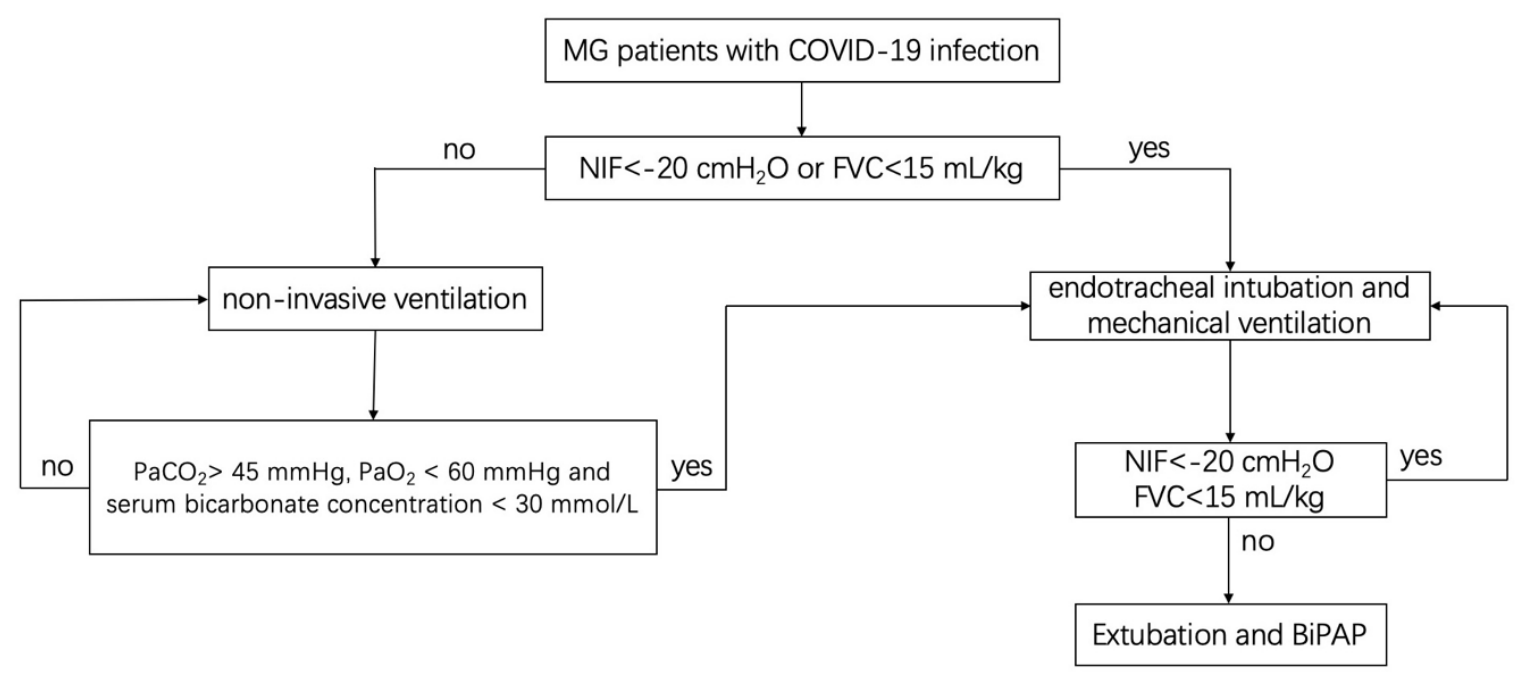

Figure 1: respiratory management in MG patients with COVID-19 infection. $\mathrm{NIF}=$ Negative inspiratory force, $\mathrm{FVC}=$ forced vital capacity.

Figure 1: Respiratory management in MG patients with COVID-19 infection. 